\title{
Pointes foliacées anciennes
}

\author{
Par J.-L. Baudet, Paris \\ Avec 5 fig.
}

$\mathrm{Z} u s$ a m menfassung. Das Problem der Blattspitzen, die verschiedentlich schon in einem technisch hochentwickelten Acheuléen auftreten, ist augenblicklich ein akutes Thema der prähistorischen Wissenschaft. Ihr Aussehen läßt in der Tat vermuten, daß es sich hierbei um eventuelle Vorläufer der Typen des Solutréen handelt. Diese Industrieformen tauchen in gewissen Zentren auf, die vielleicht am Ursprung der Solutréencivilisation stehen. $\mathrm{Da}$ aber bis heute noch keine direkte Ableitung mit Sicherheit hat herausgestellt werden können, verdienen alle weiteren Auskünfte zusammengetragen und zur Kenntnis gebracht zu werden.

Diesbezügliche Beobachtungen sind vor kurzem in stratigraphisch eindeutiger Lage im Tal der Aisne (Frankreich) - Abb. 5 - gemacht worden sowie ein interessanter Fund im südlichen Luxemburg (Gutland) - Abb. 1. Diese verhältnismäßig schmalen, langgezogenen Formen scheinen den südlichen Typen (Var) näher zu stehen als den aus Zentraleuropa bekannten Beispielen (Bayern, Tschechoslovakei).

$\mathrm{R}$ é $s$ u mé. La question des instruments lithiques foliacés, qui se remarquent parfois dans l'Acheuléen évolué, est un fait d'actualité dans les recherches préhistoriques. Leur aspect suggère effectivement la probabilité d'une préfiguration des spécimens solutréens; formule apparaissant dans certains centres qui peuvent être ̀̀ l'origine de cette dernière civilisation. C'est la raison pour laquelle, en l'absence de filiation directe parfaitement établie, tous les renseignements connus méritent d'être réunis.

Des observations récentes ont été faites, en stratigraphie, dans la vallée de l'Aisne (France) - fig. 5 - et une découverte remarquable dans le Sud du Luxembourg (Gutland) - fig. 1. Ces formes, relativement élancées, semblent plus proches des types méridionaux (Var) que des exemples déjà mentionnés en Europe centrale (Bavière, Tchécoslovaquie).

L'Abbé Breuil, dans une étude en cours de publication, résume l'essentiel des connaissances sur l'apparition des formes lithiques foliacées et l'origine, ou "les origines", du Solutréen. L'illustre préhistorien considère que ces problèmes sont loin d'être résolus, malgré l'abondance des matériaux accumulés, et qu'ils demandent l'apport d'une forte documentation supplémentaire. Par conséquent, nous croyons faire oeuvre utile en signalant des trouvailles légèrement dissemblables mais qui offrent l'avantage d'appartenir à des contrées sensiblement voisines, relativement septentrionales et encore dépourvues d'indices de ce genre.

La première est celle d'un instrument qui nous fut aimablement soumis par M. M. Heuertz, Directeur du Musée d'Histoire Naturelle de Luxembourg. Il fut recueilli dans la région S. E. du Grand-Duché - dans le Gutland - à l'altitude de $350 \mathrm{~m}$ sur les hauteurs voisines d'Osweiler. Cette découverte est due à la perspicacité de M. Charles RIPPERT (fig. 1).

Du fait même des conditions de la trouvaille, en surface du sol, son niveau stratigraphique originel est inconnu et sa position chronologique fort discutable. Néanmoins, sa similitude typologique avec certains documents palethniques d'Europe Centrale et des analogies avec les types décrits par Mlle. G. Freund et le Professeur L. Zotz, dans l'inventaire du désormais célèbre gisement bavarois du W e in b e rg, permettent de présumer une antiquité assez reculée dans le dernier glacial.

On y retrouve en effet, outre la forme, l'élégance et le peu d'épaisseur des exemples précités, le travail encore moustéroïde du façonnement. Plus encore, la pièce d'Osweiler présente une sorte de cran marginal, proximal. Particularité que l'on rencontre également à Mauern et à Předmost. Il paraît aussi très intéressant de faire remarquer que cet objet est nettement plus élancé que les formes orientales et que, sous cet aspect, il se rapproche plus ouvertement des types de la couche B II de la Baume-Bonne. Grotte-abri 


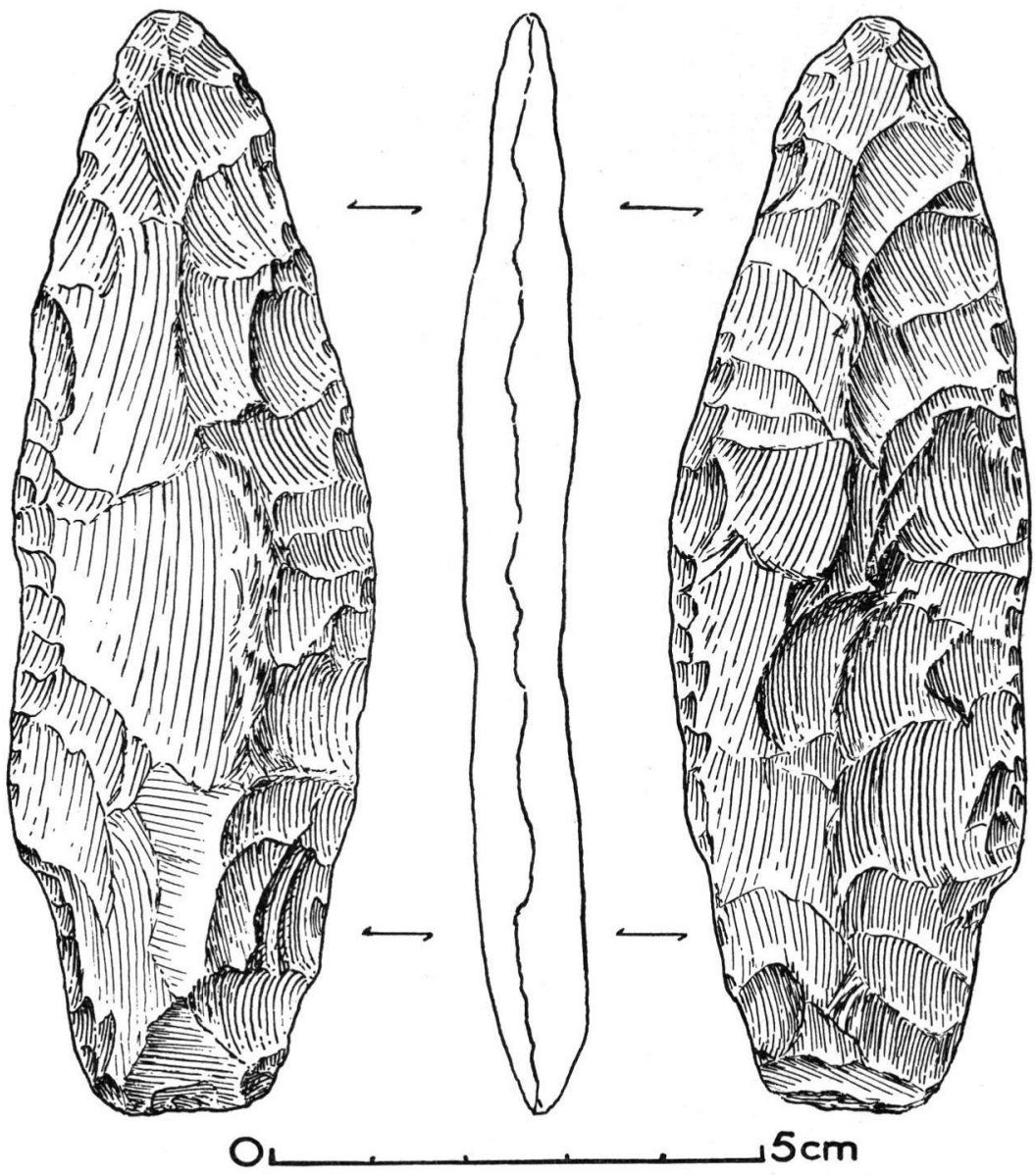

Fig. 1. Instrument foliacé. Osweiler (Grand-Duché de Luxembourg).

fouillée par B. Bоттет à Quinson (Basses-Alpes). Dans ce sens, peut-être serait-il utile d'opérer une confrontation plus intime avec les éléments 11 et 12 de la planche VI de l'étude publiée à Monaco en 1956.

Quelque soit l'importance des indices tirés des précédentes comparaisons, l'objet du Luxembourg, lié ultérieurement à d'autres jalons plus nombreux, mérite de figurer sur la liste des éléments précieux, situés sur le chemin des découvertes danubiennes à l'épanouissement solutréen français. De plus, il est opportun de signaler les caractères, aux ressemblances plus méridionales, qui l'éloignent partiellement du "Présolutréen" classique.

Nous profitons de l'occasion pour rappeler que cette dernière nomenclature est amicalement controversée par l'Abbé BreuiL, le "Présolutréen" étant l'un des premiers termes utilisés dans la bataille de l'Aurignacien (1907-1909). Notre Maître préconise l'emploi de "Weinbergien" qui, somme toute, rend un hommage encore plus vibrant aux éminents travaux effectués sur le site du bras fossile du Danube.

Notre description d'éléments susceptibles de participer à la solution d'une énigme intéressante n'est pas close. En effet, elle se complète de données qui concernent plus spécialement notre pays; ces renseignements étant d'autant plus importants qu'ils se rapportent à une époque jusqu'alors admise comme plus ancienne. 


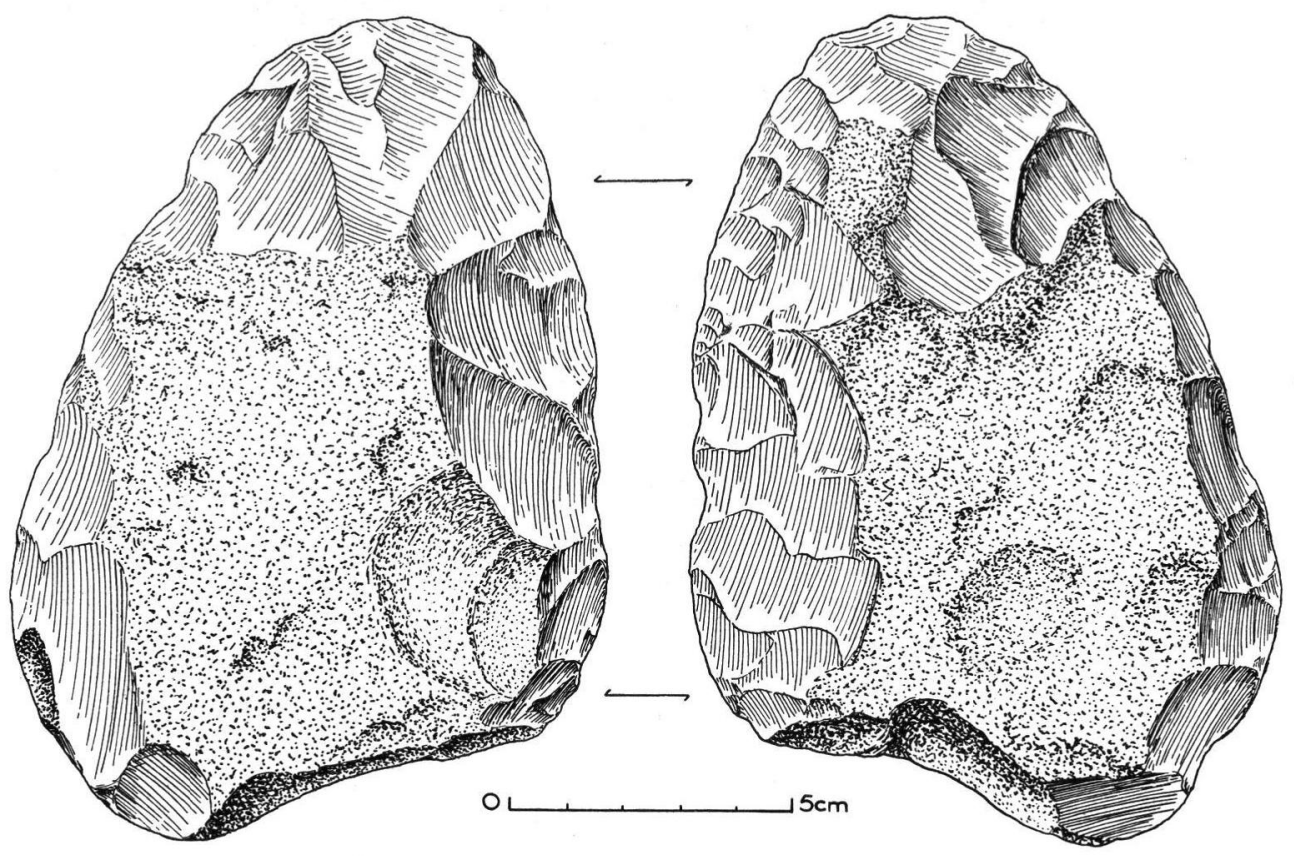

Fig. 2. Biface sur plaquette siliceuse. Pommiers, Aisne (France).

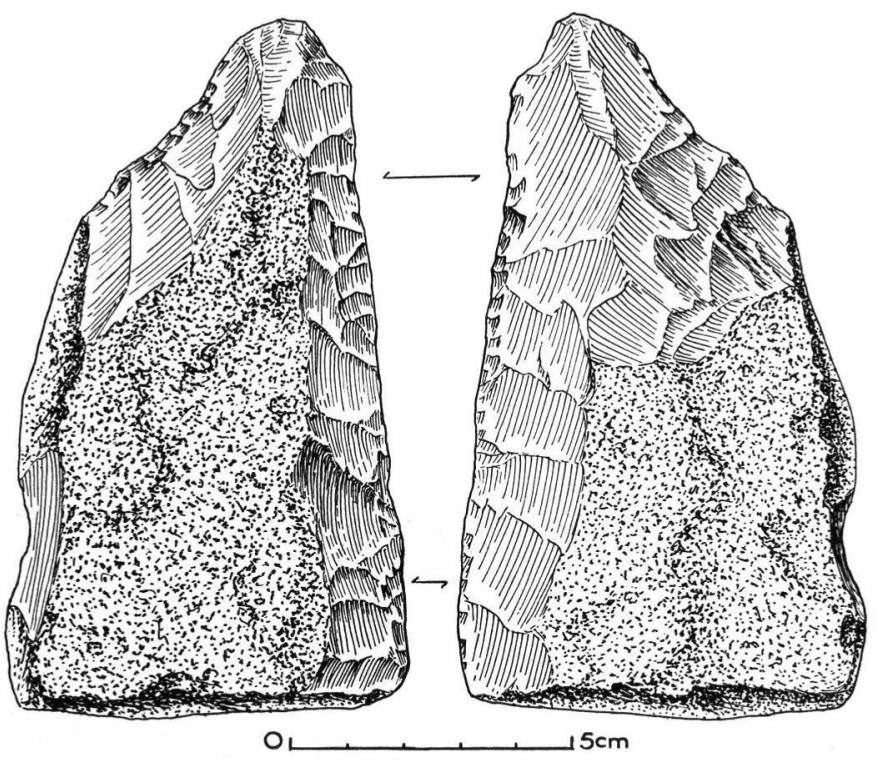

Fig. 3. Outil bifacial sur plaquette gréseuse. Soissons, Aisne (France).

Il s'agit d'indications partant d'une zone du remplissage sédimentaire de la vallée de l'Aisne, étudiée principalement aux environs de Soissons et dans le tronçon amont vers Neufchâtel, et aboutissant aux éléments préhistoriques qu'elle contient. Cet horizon - situé en bas-niveau - sableux, glauconifère, constitué de matériaux empruntés au Tertiaire, forme un lit presque ininterrompu qui s'observe parallèlement à la rivière. Il 


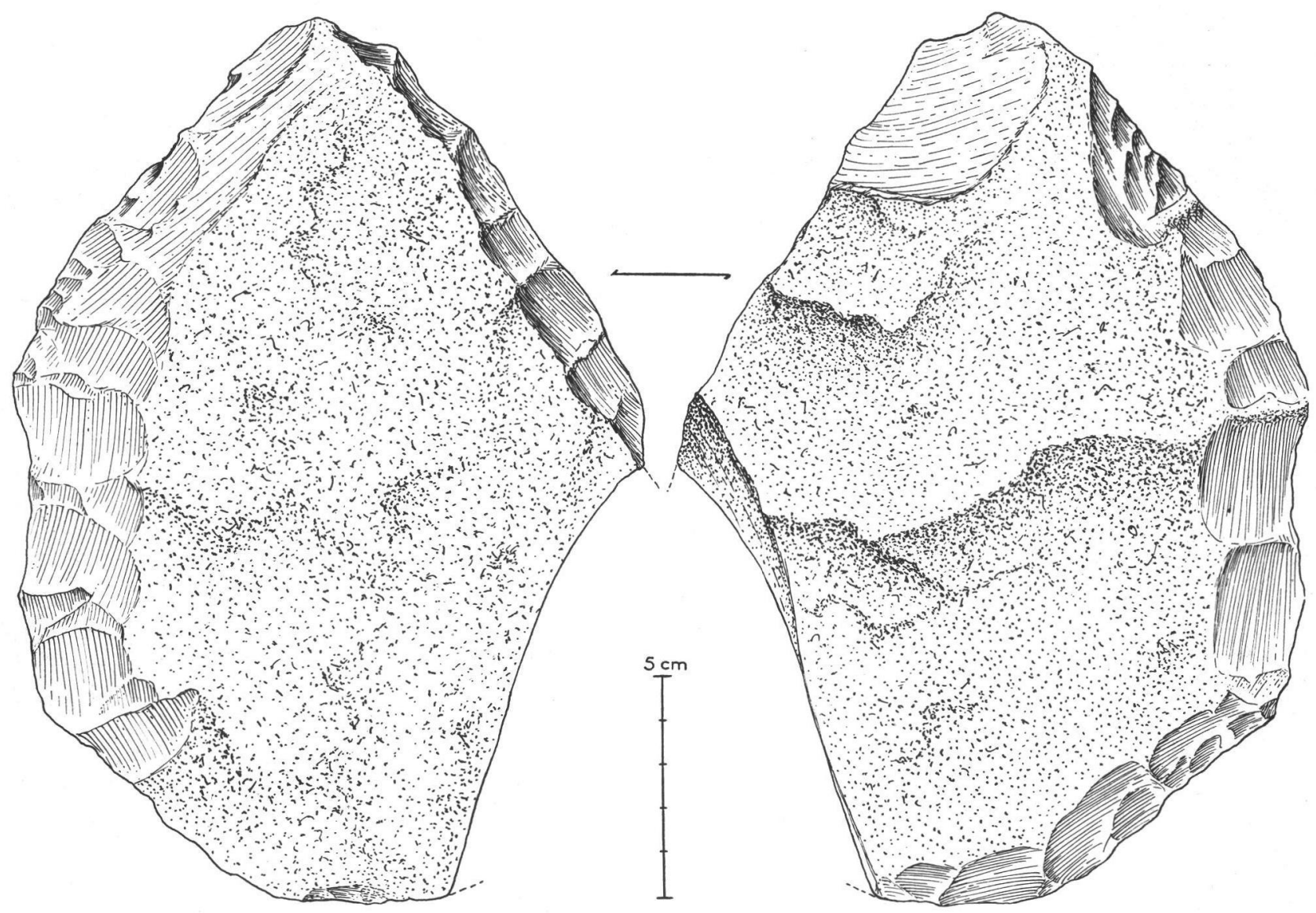

Fig. 4. Instrument discoïde sur plaquette de grès. St.-Mard, Aisne (France). 


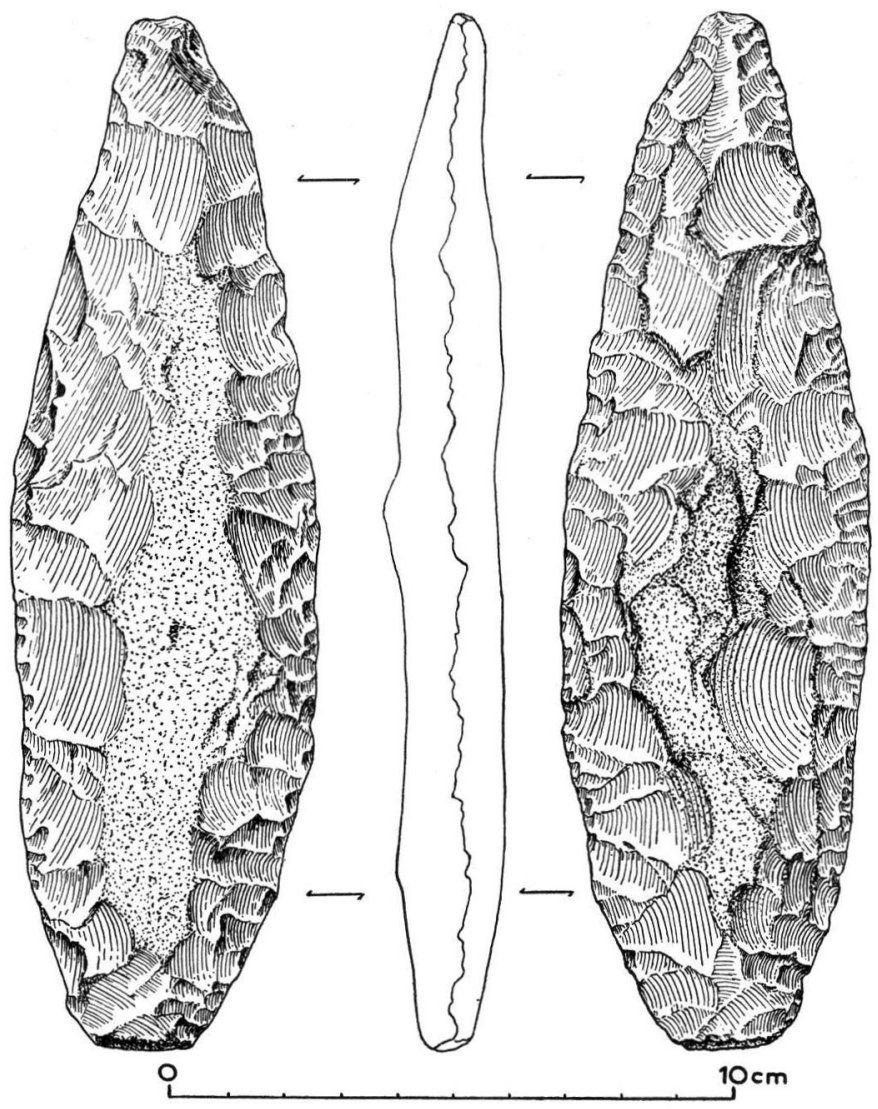

Fig. 5. Instrument foliacé. Pommiers, Aisne (France).

est inclus dans les sédiments latéraux, à quelques mètres de profondeur, dans une position nettement inférieure au thalweg actuel. Cette couche contient des bifaces tirés de plaquettes siliceuses ou gréseuses dont la plupart des spécimens ont été obtenus par simple épannelage marginal, le matériau originel étant fréquemment trop mince pour nécessiter un travail bifacial plus étendu (fig. 2 et 3 ).

Le même niveau, dont l'analyse morphoscopique des éléments quartzeux révèle un brassage fluviatile assez prononcé (nombreux polis luisants), fournit aussi des disques épannelés en bordure marginale (fig. 4). Formes déterminées, comme les bifaces ci-dessus décrits, par l'utilisation du matériau autochtone.

Mais ceci n'est pas tout. On y découvrit également plusieurs pièces foliacées - maintenant réparties dans diverses collections - dont un exemplaire nous a été généreusement confié, pour étude, par le R. P. Chrétien de Pontoise. Objet trouvé par M. Becker de Soissons et destiné aux séries du Musée de l'Homme (fig. 5). Cet instrument remarquable, de $18 \mathrm{~cm}$ de long sur une largeur maximum de $55 \mathrm{~mm}$, n'a guère plus de $18 \mathrm{~mm}$ d'épaisseur à l'endroit où le cortex conservé présente une légère protubérance. Malgré les restes de pellicule hydratée de la plaquette originelle, on remarque un travail où les enlèvements d'esquilles subparallèles sont assez fréquents et préfigurent les procédés leptolithiques.

Dans l'ensemble, la technique employée est nettement plus évoluée que celle pratiquée en surface de la pointe luxembourgeoise. De plus, cette exécution a eu comme conséquence de donner une forme très élancée qui contraste des spécimens weinbergiens. Par son 
rapport de l'intersection longueur-largeur $9 \times 20$ on y trouve un indice proche de certains exemples du Solutréen moyen classique.

Nous sommes évidemment loin de cette gamme solutréenne; mais, tant par ses offinités typologiques que par sa position stratigraphique - dans un interstade du remplissage de basse-terrasse - et par sa fraîcheur physique, nous considérons cet instrument (ainsi que ceux de même provenance) comme appartenant au dernier glacial; probablement à une phase postérieure à celle de Mauern. Evidemment, c'est une chose à revoir stratigraphiquement en détail, avec toute l'objectivité désirée, car ces indices sont indubitablement dignes d'intérêt.

La contrainte vraisemblable du matériau sur l'exécution humaine, dont nous avons à maintes reprises cité l'exemple'), trouve ici, comme dans l'outillage de la Klause (Bavière), un écho encore inédit. Mais, nous restons convaincu que, dans la vallée de l'Aisne, le niveau subcontinu de sable partiellement roulé par l'élément liquide - dans un cours à débit lent - appartient à une phase tardive du Würm $=$ Weichsel, et que cette magnifique pointe foliacée se rapporte, par conséquent, à une époque plus proche du Paléolithique supérieur que la civilisation weinbergienne. Il faut évidemment exclure tous les documents palethniques diversement répartis dans les autres horizons de remplissage du chenal, éléments repris par solifluxion ou lixiviation à des formations plus anciennes.

Nous croyons indispensable de faire connaître les quelques renseignements précités afin qu'ils puissent contribuer à l'éclaircissement de divers problèmes non encore résolus. Et, il nous a semblé particulièrement favorable, grâce à l'amabilité du Professeur WolDSTEDT, d'en donner une expression première au pays où les "Blattspitzen" ont, depuis longtemps déjà, suscité de précieuses investigations.

\section{Bibliographie récente:}

Breuil, H.: Le Solutréen. Définition. Historique. Classification. - Sous presse.

Baudet, J.-L.: Questions d'actualité. - "Revue Anthropologique", nouv. série, 1, pp. 113-123, Paris 1955. - - Façiès et matériau. - Ibidem 3, pp. 178-192, 1957.

Bottet, B.: La grotte-abri de la Baume-Bonne à Quinson (B.-A.) - "Bull. Musée d'Anthropologie préhist. Monaco“ 3, pp. 79-121, 1956.

Freund, G.: Die Blattspitzen des Paläolithikums in Europa. - Quartär-Bibliothek, Bd. 1, 349 pp., Bonn 1952.

Zoтz, L.: Das Paläolithikum in den Weinberghöhlen bei Mauern. - Quartär-Bibliothek, Bd. 2, 330 pp., Bonn 1955. - - Kösten, ein Werkplatz des Praesolutréen in Oberfranken. - QuartärBibliothek, Bd. 3, 145 pp., Bonn 1959.

Manuskr. eingeg. 18. 8. 1960.

Anschrift des Verf.: J.-L. Baudet, Professeur à l'Ecole d'Anthropologie, Institut de Paléontologie Humaine, Paris XIIIe 1, rue René Panhard.

1) a - Société d'Ethnographie de Paris, Mai 1960, b - Revue Anthropologique, 1957. 